Europhysics Letters

PREPRINT

\title{
Concentration dependence of the transition temperature in metallic spin glasses
}

\author{
R. Serral Gracià ${ }^{1}(*)$, Th. M. Nieuwenhuizen ${ }^{1}\left(^{* *}\right)$ and I. V. Lerner ${ }^{2}\left(^{* * *}\right)$ \\ 1 University of Amsterdam, Institute for Theoretical Physics - \\ Valckenierstraat 65, 1018XE Amsterdam, The Netherlands \\ 2 School of Physics and Astronomy, University of Birmingham - \\ Edgbaston, Birmingham B152TT, United Kingdom
}

PACS. 75.10.Nr - Spin-glass and other random models.

PACS. 75.50.Lk - Spin glasses and other random magnets.

\begin{abstract}
The dependence of the transition temperature $T_{g}$ in terms of the concentration of magnetic impurities $c$ in spin glasses is explained on the basis of a screened $R K K Y$ interaction. The two observed power laws, $T_{g} \sim c$ at low $c$ and $T_{g} \sim c^{2 / 3}$ for intermediate $c$, are described in a unified approach.
\end{abstract}

Metallic spin glasses such as $\mathrm{Cu}_{1-c} \mathrm{Mn}_{\mathrm{c}}, \mathrm{Ag}_{1-\mathrm{c}} \mathrm{Mn}_{\mathrm{c}}, \mathrm{Au}_{1-\mathrm{c}} \mathrm{Fe}_{\mathrm{c}}$ are alloys formed by magnetic impurities embedded in a noble metal. The transition temperature of such materials depends on the magnetic impurity concentration $c$. Different phenomena dominate for different concentrations. The mutual interaction between magnetic impurities is mediated by electrons, the $R K K Y$ interaction. It can be understood as follows: the sea of electrons interact with an impurity and the scattered wave interferes with the incoming one. This creates a pattern of spin polarizations that brings an oscillatory behaviour and a $1 / r^{3}$ fall off of the form $J(\boldsymbol{r})=A \cos \left(2 k_{F} \boldsymbol{r}\right) / \boldsymbol{r}^{3}$ at $T=0$. For very low concentrations, less than $\approx 50 \mathrm{ppm}$, the interaction can be neglected and the magnetic impurities act independently bringing the Kondo effect. For larger concentrations though less than $\approx 10$ at.\% the $R K K Y$ interaction is the dominant interaction and the spin glass phase appears. The oscillatory nature of the interaction and the position randomness of the impurities form a disordered magnet. Above this concentration, the chance of having a significant amount of impurities as first or second neighbours is high, and consequently clusters are formed. For even larger concentrations the percolation limit is reached and ferromagnetism or antiferromagnetism, depending on the type of magnetic impurities, appears. For a review on these different regimes see Ref. [1].

The spin glass region of concentrations (excluding the cluster region) exhibits two different behaviours. On one hand, for concentrations lower than $\approx 1 / 2$ at.\%, the data points approach a linear curve, $T_{g} \propto c$, while on the other hand, for higher concentrations, up to $\approx 10-15$ at.\% the fit turns to $T_{g} \propto c^{2 / 3}$. In this letter we explain these scaling laws in a unified treatment.

\footnotetext{
$\left(^{*}\right)$ E-mail: rubeng@science.uva.nl

$(* *) \quad$ nieuwenh@science.uva.nl

$(* *) \quad$ ivl@th.ph.bham.ac.uk
}

(C) EDP Sciences 
The key point lies in the fact that the $R K K Y$ interaction is not infinite ranged. At finite temperature, phonons interact with the electron sea smearing out the spin polarization pattern at large distances, consequently the interaction is cut off at a length $\Lambda_{T}$. It has the form

$$
J(\boldsymbol{r})=A e^{-r / \Lambda_{T}} \cos \left(2 k_{F} \boldsymbol{r}\right) / \boldsymbol{r}^{3}
$$

For pure metals this cut off is the thermal coherence length $\Lambda_{T}=\hbar v_{F} \beta / \pi \propto 1 / T$. For disordered metals where non-magnetic impurities exist alongside with magnetic ones, the former start to play a role [2]: the electron wave that scatters from the magnetic impurity diffuses around the non-magnetic ones before it reaches another magnetic impurity. The contributions of all such diffusive paths add coherently as long as the distance between the magnetic impurities is smaller than the thermal coherence length $\Lambda_{T}$. This leads to cutting off the typical value of the interaction [2] as in eq. (11), but with $\Lambda_{T}=(D \hbar \beta / \pi)^{1 / 2} \propto 1 / T^{1 / 2}$, where $D=v_{F}^{2} \tau / 3$ is the diffusion constant of an electron in a disordered metal and $\tau$ is the mean free time for elastic scattering. Thus for $T \tau \lesssim 1$ (assuming also that the elastic scattering dominates over the inelastic, $\tau \lesssim \tau_{\text {inel }}$ ) the effective range of the interaction for a disordered metal is shorter than for the pure case. This defines a limit between the two situations that can bring differences in the large concentration regime for certain materials since the above inequalities may well be reached in some cases.

Shegelski and Geldart [3] have derived the range of an indirect-exchange interaction in disordered metals which takes into account the $R K K Y$ interaction and sd scattering. They could well describe a wide range of experiments by fitting new length scales that appear in the problem. We shall not aim at fitting the data but to give the basic mechanism. We focus on the case where the concentration of magnetic impurities is changed with no other added impurities. Our approach does not have adjustable parameters.

We use a Hamiltonian that takes into account the fact that the magnetic impurities, i.e. "spins", are present in some sites of the lattice and not in all of them [4,5]

$$
\mathcal{H}(s)=-\frac{1}{2} \sum_{\boldsymbol{r}, \boldsymbol{r}^{\prime}} J\left(\boldsymbol{r}-\boldsymbol{r}^{\prime}\right) s_{\boldsymbol{r}} s_{\boldsymbol{r}^{\prime}} c_{\boldsymbol{r}} c_{\boldsymbol{r}^{\prime}}-H \sum_{\boldsymbol{r}} s_{\boldsymbol{r}} c_{\boldsymbol{r}}
$$

where $s_{\boldsymbol{r}}$ represents the spin on site $\boldsymbol{r}$ and $c_{\boldsymbol{r}}=1,0$ whether a spin is present on site $\boldsymbol{r}$ or not. $J\left(\boldsymbol{r}-\boldsymbol{r}^{\prime}\right)$ is the $R K K Y$ interaction between sites $\boldsymbol{r}$ and $\boldsymbol{r}^{\prime}$. This model is used since it contains from the beginning a dependence on the concentration via an average of the $c_{\boldsymbol{r}}$. It is a random site problem, since the randomness comes from the distribution of spins in a lattice, and not a random bond problem where each bond has a random strength, as is for example for the SK model. This model can be solved in the low concentration limit via a mean field approximation in the replica scheme [4,5]. The transition temperature was found to satisfy the following condition

$$
c \sum_{\boldsymbol{r}} \tanh ^{2}[\beta J(\boldsymbol{r})]=1
$$

where $\boldsymbol{r}$ represents each of the sites of the lattice since the factors $c_{r}$ have been taken in average. Combining the $R K K Y$ interaction in eq. (1) with eq. (3) we basically have an equation of the form

$$
4 \pi c \int_{0}^{\Lambda_{T}} \mathrm{~d} r r^{2} \tanh ^{2}\left[\frac{A \beta}{r^{3}}\right]=1
$$

where the upper limit of the integral accounts for the interaction cut off, and, as a first approximation, we can consider that the oscillations of the cosine are not of qualitative importance 
in the range where the hyperbolic tangent squared has significant values. Eq. (4) gives the transition temperature $T_{g}$ for a given concentration $c$. For small concentrations, in order to satisfy the equation, the transition temperature has to be small, which allows us to extend the upper limit of the integral to infinity. It yields

$$
4 \pi c \beta_{g} \int_{0}^{\infty} \mathrm{d} x x^{2} \tanh ^{2}\left[\frac{A}{x^{3}}\right]=1
$$

which leads to the expected result [4, 5

$$
T_{g} \propto c
$$

For larger concentrations, the transition temperature has to decrease. Then the upper limit remains finite and the $\tanh ^{2}$ in eq. (4) can be approximated by 1 . We get

$$
4 \pi c \int_{0}^{\Lambda_{T}} \mathrm{~d} r r^{2}=c \frac{4 \pi}{3} \Lambda_{T}^{3}=1
$$

From the middle expression we see that the transition takes place when there starts to be on the average more than one impurity in the range of attraction of the $R K K Y$ interaction, as one might have expected. Since for a disordered metal $\Lambda_{T} \propto T^{-1 / 2}$, the scaling law then reads

$$
T_{g} \propto c^{2 / 3}
$$

We want to stress that the $2 / 3$ power law is intimately related to the fact that the system is considered to be a disordered metal, having $\Lambda_{T} \propto T^{-1 / 2}$. In this sense, we consider metals with a small amount of non-magnetic impurities (for a study on the effect of a variation on the concentration of non-magnetic impurities see Refs. 3 6] ). For a pure metal, i.e. for $T_{g} \tau \gg 1$, where the range of the $R K K Y$ interaction is proportional to the inverse of the temperature, $\Lambda_{T} \propto T^{-1}$, the scaling law in eq. (8) becomes $T_{g} \propto c^{1 / 3}$. In the intermediate case, i.e. for $T_{g} \tau \sim 1$, to consider the system as being disordered is not anymore a good approximation, a fact that may bring values of the exponent $\phi$ lower than the $2 / 3$ predicted for the disordered case, i.e. for $T_{g} \tau \ll 1$. An example of that is the case for $\mathrm{Au}_{1-\mathrm{c}} \mathrm{Fe}_{\mathrm{c}}$ where the exponent $\phi \approx 0.58$ [7.

We have explained here the two "pure" scaling laws $T_{g} \propto c$ and $T_{g} \propto c^{2 / 3}$ (or $T_{g} \propto c^{1 / 3}$ for pure metals) corresponding to the canonical spin glass [1]. These are the limiting situations and effective exponents found in experiments may lie between $1 / 3$ and 1 . We suspect that in the experiments performed till now, a non-negligible amount of non-magnetic impurities were always present in the sample. We therefore propose to perform new refined experiments in order to test the presence of lower exponents $\phi$.

This work is part of the research programme of the 'Stichting voor Fundamenteel Onderzoek der Materie (FOM)', which is financially supported by the 'Nederlandse Organisatie voor Wetenschappelijk Onderzoek (NWO)'. Th.M.N. acknowledges hospitality at the University of Birmingham. 
EUROPHYSICS LETTERS

\section{REFERENCES}

[1] Mydosh, J. A., Spin Glasses. An experimental introduction (Taylor \& Francis) 1993.

[2] Zyuzin A. Y. and Spivak B. Z., JETP Lett., 43 (1986) 234; Bulaevskit, L.N. and Panyukov S. V., ibid 240; Bergmann, G., Phys. Rev. B, 36 (1987) 2469

[3] Shegelski, M. R. A. and Geldart, D. J. W., Phys. Rev. B, 46 (1992) 2853; 46 (1992) 5318.

[4] Nieuwenhuizen, Th. M., Europhys. Lett., 24 (1993) 797.

[5] Nieuwenhuizen, Th. M. and van Duin, C. N. A., Eur. Phys. B, 7 (1999) 191.

[6] Vier, D. C. and Schultz, S., Phys. Rev. Lett., 54 (1985) 150.

[7] Cannella, V. and Mydosh, J. A., Phys. Rev. B, 6 (1972) 4220. 